\title{
Electronic structure of new iron-based superconductors: from pnictides to chalcogenides and other similar systems
}

\author{
${ }^{a}$ I. A. Nekrasov ${ }^{1)},{ }^{a, b}$ M. V. Sadovskii ${ }^{2)}$ \\ ${ }^{a}$ Institute for Electrophysics, Russian Academy of Sciences, Ural Branch, Amundsen str. 106, Ekaterinburg, 620016, Russia \\ ${ }^{b}$ Institute for Metal Physics, Russian Academy of Sciences, Ural Branch, S. Kovalevskaya str. 18, Ekaterinburg, 620990, Russia
}

\begin{abstract}
This review discusses and compares electronic spectra of new iron-based high-temperature superconductors (HTSC), as well as a number of chemically similar compounds. Particular attention is payed to iron chalcogenide $\mathrm{K}_{1-x} \mathrm{Fe}_{2-y} \mathrm{Se}_{2}$, which is isostructural to $\mathrm{BaFe}_{2} \mathrm{As}_{2}$ (122) pnictide. It is shown, that Fermi surfaces of $\mathrm{K}_{1-x} \mathrm{Fe}_{2-y} \mathrm{Se}_{2}$ are essentially different from those for pnictides. Using LDA+DMFT and LDA'+DMFT calculations we show, that electronic correlations in $\mathrm{K}_{1-x} \mathrm{Fe}_{2-y} \mathrm{Se}_{2}$ influence the electronic structure much more significantly, than in the most studied 122 system. We also discuss the electronic structure of several multiple-band superconductors, chemically similar to iron-based HTSC, with relatively small values of $T_{c}$, such as $\mathrm{SrPt}_{2} \mathrm{As}_{2}, \mathrm{APt}_{3} \mathrm{P},(\mathrm{Sr}, \mathrm{Ca}) \mathrm{Pd}_{2} \mathrm{As}_{2}$, and non superconducting compound $\mathrm{BaFe}_{2} \mathrm{Se}_{3}$. It is shown, that electronic structure of these systems is very different from previously studies iron pnictides and chalcogenides. The value of $T_{c}$ in these systems can be understood within the simple BCS model.
\end{abstract}

'PACS: 71.20.-b, 71.27.+a, 74.20.Fg, 74.25.Jb, 74.70.-b

\section{INTRODUCTION}

The discovery of new class of iron-base supercon'ductors [1] attracted great attention, leading to outstanding upsurge of both experimental and theoretical 'works (cf. reviews [2, 3, 4]). The known at the present moment iron-based superconductors can be divided in two classes: pnictides and chalcogenides. Below we list the typical representatives of these classes, currently un'der study (detailed references on experimental works can be found in [5]):

1. Doped RE1111 systems ( $R E=L a, C e, P r, N d, S m, T b, D y)$ with superconducting transition temperature $\mathrm{T}_{c}$ of the order of $25-55 \mathrm{~K}$, with chemical formula $\mathrm{RE} \mathrm{O}_{1-x} \mathrm{~F}_{x} \mathrm{FeAs}$;

2. Doped A122 systems $(\mathrm{A}=\mathrm{Ba}, \mathrm{Sr})$, such as $\mathrm{Ba}_{1-x} \mathrm{~K}_{x} \mathrm{Fe}_{2} \mathrm{As}_{2}$ with $\mathrm{T}_{c}$ of the order of $38 \mathrm{~K}$;

3. The so called 111 systems like $\mathrm{Li}_{1-x} \mathrm{FeAs}$ with $\mathrm{T}_{c} \sim 18 \mathrm{~K}$

4. (Sr,Ca,Eu)FFeAs with $\mathrm{T}_{c} \sim 36 \mathrm{~K}$;

5. $\mathrm{FeSe}_{x}, \mathrm{FeSe}_{1-x} \mathrm{Te}_{x}$ with $\mathrm{T}_{c}$ up to 14 ;

6. (K,Cs $)_{1-x} \mathrm{Fe}_{2-y} \mathrm{Se}_{2}$ systems with $\mathrm{T}_{c}$ up to $31 \mathrm{~K}$.

\footnotetext{
2) E-mail: nekrasov@iep.uran.ru

2) E-mail: sadovski@iep.uran.ru
}

Following the discovery of HTSC in iron arsenides the intensive search of new systems has lead to the discovery of several new superconducting systems, which can be considered as chemical analogs of iron pnictides and iron chalcogenides, e.g. such as $\mathrm{BaNi}_{2} \mathrm{As}_{2}$ [6], $\mathrm{SrNi}_{2} \mathrm{As}_{2}$ [7], $\mathrm{SrPt}_{2} \mathrm{As}_{2}$ [8], SrPtAs [9]. However, these systems possess rather low superconducting transition temperature $T_{c}$. Recently, a number of new platinum based systems were also discovered: $\mathrm{APt}_{3} \mathrm{P}$ (A=Sr,Ca,La) [10] with $T_{c}$ equal to $8.4 \mathrm{~K}, 6.6 \mathrm{~K}$ and $1.5 \mathrm{~K}$ correspondingly. Of interest is also the $\mathrm{BaFe}_{2} \mathrm{Se}_{3}$ system (Ba123) [11], which was initially considered as potential superconductor similar to (K, Cs) $\mathrm{Fe}_{2} \mathrm{Se}_{2}$ (preliminary data has shown here superconductivity with $T_{c} \sim 11 \mathrm{~K}$ ). However, later it was shown, that in Ba123 superconductivity is not observed up to $1.8 \mathrm{~K}[12]$. This system was shown to be an antiferromagnetic (AFM) "spin ladder" with nontrivial magnetic ordering [11, 12]. Superconductivity was also discovered in palladium compounds: ( $\mathrm{Sr}, \mathrm{Ca}) \mathrm{Pd}_{2} \mathrm{As}_{2}$ with $T_{c} 0.92$ and 1.27 correspondingly [13].

This short review is based upon the cycle of our investigations of electronic structure of all these systems within the framework of density functional theory and modern methods to take into account strong correlations, like LDA+DMFT [14] and $\mathrm{LDA}^{\prime}+\mathrm{DMFT}[15,16]$ for iron chalcogenides $[17,18]$, as well as upon the results of LDA calculations for above mentioned similar systems [19, 20, 21, 22].

These works were preceded by rather long series of our works on iron pnictides and chalcogenides [23, 24, 
$25,26,27]$, the results of these works were discussed in details in our previous reviews $[2,5]$

All LDA calculations of band structures presented below were performed using the basis of linearized muffin-tin orbitals (LMTO) [28], with default parameters.

We shall see, that all systems under discussion are typical multiple-band superconductors, making them interesting from the point of view of HTSC search $[29,30]$.

\section{ELECTRONIC STRUCTURE AND CORRELATIONS IN IRON CHALCOGENIDES}

\section{$21 \quad \mathrm{KFe}_{2} \mathrm{Se}_{2}$ system}

One of the most interesting iron chalcogenide systems, studied in recent years, are the compounds like $\mathrm{K}_{1-x} \mathrm{Fe}_{2-y} \mathrm{Se}_{2}$ [31], $\mathrm{Cs}_{1-x} \mathrm{Fe}_{2-y} \mathrm{Se}_{2}$ [11] and $(\mathrm{Tl}, \mathrm{K}) \mathrm{Fe}_{1-x} \mathrm{Se}_{2-y}[32]$. It was shown experimentally, that in $\mathrm{K}_{0.8} \mathrm{Fe}_{1.6} \mathrm{Se}_{2}$ nontrivial AFM ordering is observed, with pretty high Neel temperature of the order of 550 . Simultaneously, practically in the same temperature region, there is vacancy ordering in iron sublattice [33]. In fact, in $\mathrm{K}_{1-x} \mathrm{Fe}_{2-y} \mathrm{Se}_{2}$ system many different phases are observed, so that at present it is not completely clear, which phase produces superconductivity. In a number of papers it was proposed, that the generic phase for superconductivity is stoichiometric composition $\mathrm{KFe}_{2} \mathrm{Se}_{2}[5,34,35]$, though different points of view were also expressed [36]. Note, that attempts of the synthesis of stoichiometric crystal $\mathrm{KFe}_{2} \mathrm{Se}_{2}$ were up to now unsuccessful.

Important step in the studies of this iron selenide were the measurements ARPES spectra in $\mathrm{K}_{0.76} \mathrm{Fe}_{1.72} \mathrm{Se}_{2}$ [37]. According to these experiments, quasiparticle dispersions are significantly renormalized due to electron correlations. For example, the experimentally determined value of quasiparticle mass renormqalization for $\mathrm{Fe}-3 \mathrm{~d}_{x z, y z}$ orbitals is equal to 3 , while for $\mathrm{Fe}-3 \mathrm{~d}_{x y}$ orbital it is equal to 10 . These facts clearly require theoretical analysis of electron - electron interaction effects upon properties of this system.

For the first time, the electronic spectrum of $\mathrm{K}_{1-x} \mathrm{Fe}_{2} \mathrm{Se}_{2}$ in LDA approximation was obtained in Refs. [27, 38].

Crystal structure.

As we noted above, $\mathrm{K}_{1-x} \mathrm{Fe}_{2} \mathrm{Se}_{2}$ and $\mathrm{Cs}_{1-x} \mathrm{Fe}_{2} \mathrm{Se}_{2}$ systems are structural analogs of Ba122 (cf. Ref. [24]) with following crystal structure parameters: $\mathrm{K}_{1-x} \mathrm{Fe}_{2} \mathrm{Se}_{2}-a=3.9136 \AA$ and $c=14.0367 \AA$ and

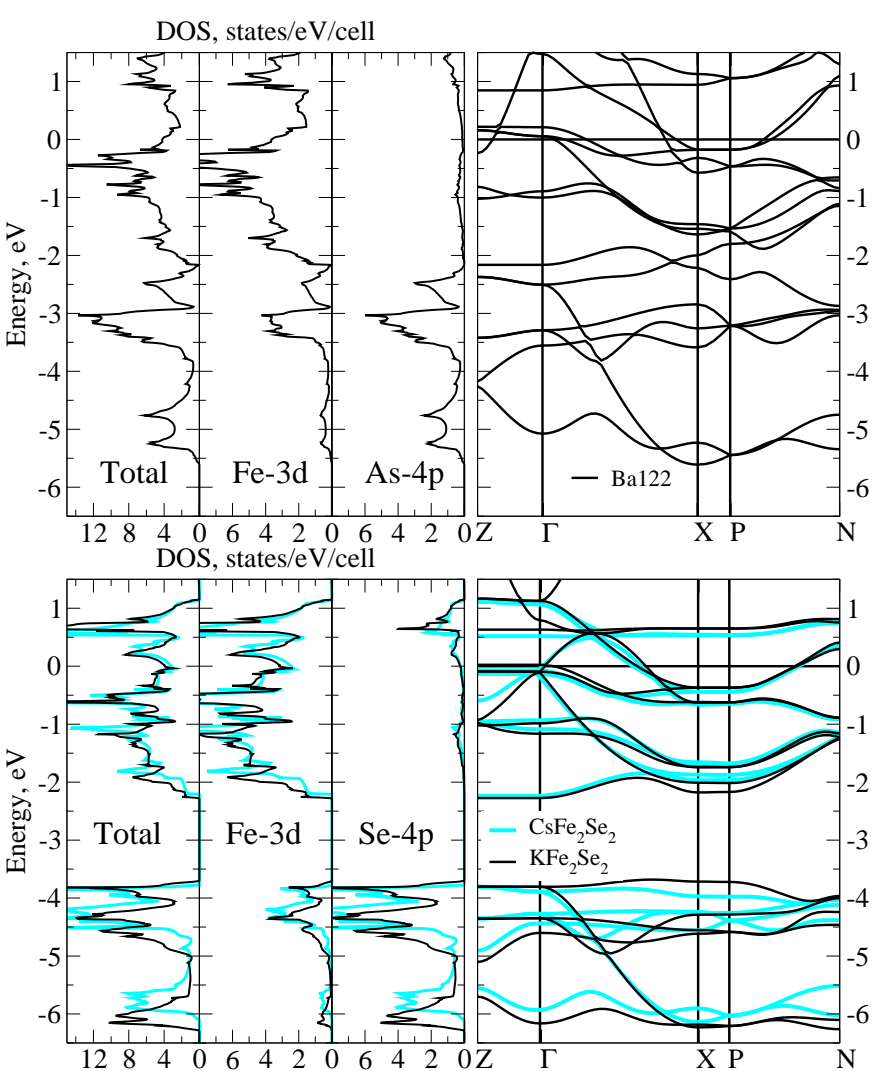

Fig. 1. Band dispersions and densities of states for Ba122 (left), $\mathrm{KFe}_{2} \mathrm{Se}_{2}$ (black lines) and $\mathrm{CsFe}_{2} \mathrm{Se}_{2}$ (gray lines)(right), obtained in LDA calculations. Fermi level is at zero energy.

$z_{S e}=0.3539 \quad[31] ; \quad \mathrm{Cs}_{1-x} \mathrm{Fe}_{2} \mathrm{Se}_{2} \quad-\quad a=3.9601 \AA \quad$, $c=15.2846 \AA$ and $z_{S e}=0.3439$ [11].

Electronic structure.

In Fig. 1 we compare band structures and densities of states of Ba122 [24] (left) with corresponding calculation results for $\mathrm{K}_{1-x} \mathrm{Fe}_{2} \mathrm{Se}_{2}$ (black lines) and $\mathrm{Cs}_{1-x} \mathrm{Fe}_{2} \mathrm{Se}_{2}$ (gray lines) (right) for stoichiometric compositions with $\mathrm{x}=0$ [27]. Comparing $\mathrm{K}_{1-x} \mathrm{Fe}_{2} \mathrm{Se}_{2}$ and $\mathrm{Cs}_{1-x} \mathrm{Fe}_{2} \mathrm{Se}_{2}$ systems we can note, that both have practically identical electronic structures. In contrast from Ba122 in these systems Fe-3d and Se-4p states are separated in energy. Also in $\mathrm{AFe}_{2} \mathrm{Se}_{2} \mathrm{Se}-4 \mathrm{p}$ states are 0.7 eV lower in energy, as compared to As-4p states.

Similarly to Ba122 in chalcogenides under discussion practically only Fe-3d states cross the Fermi level. Also similarly to Ba122 [24] and other iron pnictides, the main contribution to density of states at the Fermi level comes from $t_{2 g}$ states $(x y, x z y z)$. The states of $e_{g}$ symmetry $\left(3 z^{2}-r^{2}\right.$ and $\left.x^{2}-y^{2}\right)$ practically do not contribute at the Fermi level. Main difference between Ba122 and new systems is observed near the $\Gamma$-point. In $\mathrm{Z}-\Gamma$ direc- 


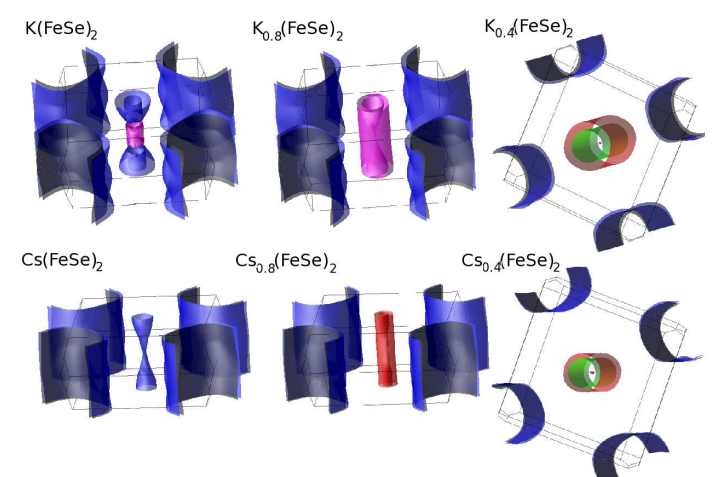

Fig. 2. Fermi surfaces obtained from LDA calculations for $\mathrm{K}_{1-x} \mathrm{Fe}_{2} \mathrm{Se}_{2}$ (above) and $\mathrm{Cs}_{1-x} \mathrm{Fe}_{2} \mathrm{Se}_{2}$ (below) for different doping levels: $\mathrm{x}=0$ - left, $\mathrm{x}=0.2$ - in the middle, and $\mathrm{x}=0.6$ - right.

tion for (K,Cs) $\mathrm{Fe}_{2} \mathrm{Se}_{2}$ systems the anti-bonding part of Se- $4 \mathrm{p}_{z}$ orbital forms an electronic pocket. In Ba122 corresponding band is $0.4 \mathrm{eV}$ higher in energy an is much steeper, so that this band passes far enough from the $\Gamma$ point. However, if we dope (K,Cs) $\mathrm{Fe}_{2} \mathrm{Se}_{2}$ by holes, we obtain the band structure in the vicinity of the Fermi level, which is similar to bands in Ba122 (at 60\% hole doping) - three hole cylinders, while the stoichiometric system $\mathrm{KFe}_{2} \mathrm{Se}_{2}$ possesses one small electronic pocket and large, hole-like pocket, while in $\mathrm{CsFe}_{2} \mathrm{Se}_{2}$ there is only one electronic pocket around the $\Gamma$-point.

In Fig. 2 we show Fermi surfaces obtained from LDA calculations for $\mathrm{K}_{1-x} \mathrm{Fe}_{2} \mathrm{Se}_{2}$ (above) and $\mathrm{Cs}_{1-x} \mathrm{Fe}_{2} \mathrm{Se}_{2}$ (below) for different doping levels: $\mathrm{x}=0-$ left, $\mathrm{x}=0.2-$ in the middle, and $\mathrm{x}=0.6$ - right. In all cases we observe two practically two-dimensional electron sheets at the corners of the Brillouin zone, independent of the doping level. Main difference in comparison to Ba122 pnictide is observed for (K,Cs) $\mathrm{Fe}_{2} \mathrm{Se}_{2}$ systems around the $\Gamma$-point, especially for $\mathrm{x}=0$ and $\mathrm{x}=0.2$. For doping level $\mathrm{x}=0.6$ both $\mathrm{K}$ and Cs selenides possess Fermi surfaces similar to those in Ba122 pnictide (cf. Fig. 2 and Ref. [24]) with typical hole-like cylinders at the center of Brillouin zone.

Thus, for doping levels corresponding to superconducting phase, the topology of Fermi surfaces is quite different from that in Ba122. In particular, there is no "nesting" of electronic and hole Fermi surfaces at all (the property of "nesting" is considered in many works on pnictides as an important property for explanation of their magnetic and superconducting properties).

In Ref. [11] it was shown that the temperature of superconducting transition $\mathrm{T}_{c}$ of $\mathrm{K}$ and $\mathrm{Cs}$ materials under study depends of the height of Se ion above Fe ions plane [39]. This dependence was studied in details in our work [40]. Let us make simple esti- mates of $T_{c}$ using the standard BCS expression for $T_{c}$ : $T_{c}=1.14 \omega_{D} e^{-2 / g N\left(E_{F}\right)}$. The value of the total density of states at the Fermi level $\mathrm{N}\left(E_{F}\right)$ for $\mathrm{KFe}_{2} \mathrm{Se}_{2}$ is equal to 3.94 states $/ \mathrm{eV} /$ cell, while for $\mathrm{CsFe}_{2} \mathrm{Se}_{2}$ it is 3.6 states $/ \mathrm{eV} /$ cell. Taking the value of Debye frequency $\omega_{D}=350 \mathrm{~K}$ and the coupling constant $g=0.21 \mathrm{eV}$, obtained for Ba122 (cf. Ref. [40]), we get $\mathrm{T}_{c}=34 \mathrm{~K}$ and 28.6K for $\mathrm{K}$ and $\mathrm{Cs}$ selenides correspondingly (the ratio of $T_{c}$ for these systems is equal to 1.18). These estimates agree rather well with experimental data of $31 \mathrm{~K}$ [31] and $27 \mathrm{~K}$ ( $T_{c}$ ratio 1.15$)$ [11]. Smaller values of $T_{c}$ in $\mathrm{CsFe}_{2} \mathrm{Se}_{2}$ can be explained by isotope effect. Taking into account that $60 \%$ hole doping leads to higher values of $\mathrm{N}\left(E_{F}\right)$ for both systems, which are (according to our calculations) 4.9 states $/ \mathrm{eV} /$ cell in $\mathrm{K}$ selenide and 4.7 states $/ \mathrm{eV} /$ cell in $\mathrm{Cs}$ selenide, we can expect, that corresponding temperatures of superconducting transition for these systems at this doping level will be correspondingly $57 \mathrm{~K}$ and $52 \mathrm{~K}$.

Note that these estimates do not necessarily assume the electron-phonon mechanism of superconductivity, and the value of $\omega_{D}$ can be referred to any Bosonic excitation, responsible for pairing, e.g. to spin fluctuations.

\section{$22 \mathbf{K}_{0.76} \mathrm{Fe}_{1.72} \mathrm{Se}_{2}$ system}

To study correlation effects in $\mathrm{K}_{0.76} \mathrm{Fe}_{1.72} \mathrm{Se}_{2}$ system [17], as well as correlation effects in $\mathrm{K}_{1-x} \mathrm{Fe}_{2-y} \mathrm{Se}_{2}$ at different hole dopings [18], we have used the standard LDA+DMFT [14] approach and its modification - $\mathrm{LDA}^{\prime}+\mathrm{DMFT}$, developed by us [15, 16], allowing consistent solution of the "double counting" problem of Coulomb interaction [41].

First of all, let us discuss the band structures for stoichiometric composition $\mathrm{KFe}_{2} \mathrm{Se}_{2}$, obtained within LDA and LDA $^{\prime}$ calculations. Similarly to the case of transition metal oxides (cf. Refs. [15, 16]) LDA' calculations for $\mathrm{KFe}_{2} \mathrm{Se}_{2}$ produce energy dispersions at the Fermi level close to those obtained within LDA. However, there are certain deviations near $\Gamma$-point. There is a shift of Se-4p band in $\mathrm{LDA}^{\prime}$ calculations down in energy with respect to LDA bands of the order of 0.5 eV (cf. Fig. 4 and Ref. [18]).

For DMFT calculations we have taken the values of Coulomb and exchange interactions at Fe-3d orbitals to be equal to $U=3.75 \mathrm{eV}$ and $J=0.56 \mathrm{eV}$, correspondingly, in agreement with Ref. [37]. inverse temperature $\beta=40 \mathrm{eV}^{-1}(290 \mathrm{~K})$, the number imaginary time intervals $L=180$ and the number of pseudospin flips $\sim 10^{6}$ to reach self-consistency. For $\mathrm{K}_{0.76} \mathrm{Fe}_{1.72} \mathrm{Se}_{2}$ the total number of electrons in DMFT calculations was taken to be $\mathrm{n}_{e}=26.52$. 
For the given doping level the account of local Coulomb interaction has lead to the following changes close to the Fermi level [17]: practically nothing has changed for Fe- $3 \mathrm{~d}_{x^{2}-y^{2}}$ orbitals, for Fe- $3 \mathrm{~d}_{3 z^{2}-r^{2}}$ states the Hubbard band appeared above the Fermi level, while for $\mathrm{Fe}-3 \mathrm{~d}_{x z, y z}$ and $\mathrm{Fe}-3 \mathrm{~d}_{x y}$ orbitals we observe the strong change in the density of states, reminding pseudogap behavior. Thus, even from the analysis of the density of states only, we can conclude, that correlation effects in $\mathrm{K}_{0.76} \mathrm{Fe}_{1.72} \mathrm{Se}_{2}$ compound are quite relevant. At the same time, their role for different orbitals may be quite different. Most important these effects are for $\mathrm{Fe}-3 \mathrm{~d}_{x z, y z}$ and Fe- $3 \mathrm{~d}_{x y}$ orbitals.

In Fig. 3 we compare spectral functions, calculated within LDA+DMFT (left side, lower figure) and $\mathrm{LDA}^{\prime}+\mathrm{DMFT}$ (right side, lower figure) with angle resolved photoemission (ARPES) spectra for different types of polarization (upper figures) [37] for chemical composition $\mathrm{K}_{0.76} \mathrm{Fe}_{1.72} \mathrm{Se}_{2}$. In the left upper part of

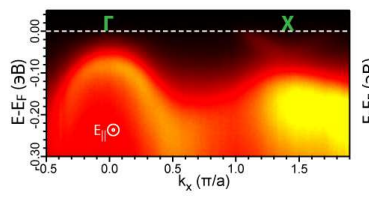

LDA+DMFT

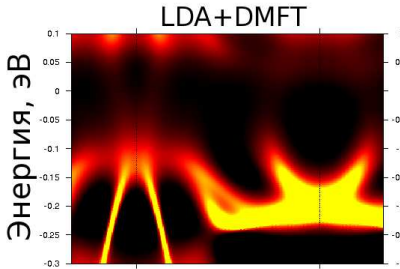

Fig. 3. Comparison of spectral functions obtained within LDA+DMFT (left side, below) and $\mathrm{LDA}^{\prime}+\mathrm{DMFT}$ (right side, below) calculations with ARPES spectrum (above) for $\mathrm{K}_{0.76} \mathrm{Fe}_{1.72} \mathrm{Se}_{2}$. Intensity of spectral function is shown by color. Zero energy is at the Fermi level.

Fig.3 we show the ARPES spectrum, obtained with light polarized along the iron layers, while in the right upper part of the figure we show the ARPES spectrum, obtained from the sum of polarizations along and across iron layers. In the lower part of Fig. 3 we show spectral functions, obtained within LDA+DMFT and $\mathrm{LDA}^{\prime}+\mathrm{DMFT}$ calculations for $\Gamma-X$-direction, studied in the experiments for the same interval of energies.

Both spectral functions and ARPES spectra show, that close to the Fermi level in energy interval \pm 0.05 $\mathrm{eV}$ there are no well-defined maxima of intensity, besides the vicinity of $\Gamma$-point in the experiments with full polarization. This fact indicates, that $\mathrm{K}_{0.76} \mathrm{Fe}_{1.72} \mathrm{Se}_{2}$ is a bad metal and confirms the pseudogap behavior, observed in the density of states [17]. High intensity region near $X$-point in ARPES spectra is present in the energy interval $(-0.25,-0.1)$, where we observe quasiparticle bands in LDA+DMFT and $\mathrm{LDA}^{\prime}+\mathrm{DMFT}^{\mathrm{D}}$ spectral functions. Close to $\Gamma$-point in one of the ARPES spectra we see the wide energy dispersion with broad maximum, while in other ARPES spectrum we observe energy dispersion with more sharp maximum, which extends up to the Fermi level. In calculated spectral densities in this energy interval we obtain two quasiparticle bands. The form of quasiparticle band with wide maximum is almost the same for the spectral functions obtained within LDA+DMFT and $\mathrm{LDA}^{\prime}+\mathrm{DMFT}$, while the form of quasiparticle band with narrow maximum in obtained $\mathrm{LDA}^{\prime}+\mathrm{DMFT}$ calculations is closer to energy dispersion observed in ARPES spectrum (cf. Fig. 3).

Thus we conclude, that for iron selenide $\mathrm{K}_{0.76} \mathrm{Fe}_{1.72} \mathrm{Se}_{2}$ correlation effects play an important role. They lead to significant changes if LDA energy dispersion. In contrast to iron arsenides, where quasiparticle bands close to the Fermi level are welldefined $[42,43,44,45]$, in $\mathrm{K}_{0.76} \mathrm{Fe}_{1.72} \mathrm{Se}_{2}$ system we observe strong suppression of quasiparticle bands at the Fermi level. This confirms that correlation effects in $\mathrm{K}_{0.76} \mathrm{Fe}_{1.72} \mathrm{Se}_{2}$ are stronger, than in iron arsenides. The value of quasiparticle renormalization (correlation narrowing) of bands at the Fermi level is equal to 4-5, while in iron arsenides this factor is equal to 2-3 [45] for the same values of interaction parameters.

As electronic properties of $\mathrm{K}_{0.76} \mathrm{Fe}_{1.72} \mathrm{Se}_{2}$ system in the vicinity of Fermi level are strongly affected by correlation effects, the question arises: how correlation effects change at different doping levels in $\mathrm{K}_{1-x} \mathrm{Fe}_{2-y} \mathrm{Se}_{2}$ compounds. To study this problem, in Ref. [18] we have chosen three doping levels: one corresponding to stoichiometric composition $\left(\mathrm{n}_{e}=29.00\right)$, and two others with total electron numbers $\mathrm{n}_{e}=28.00$ and $\mathrm{n}_{e}=27.20$. As for $\mathrm{K}_{0.76} \mathrm{Fe}_{1.72} \mathrm{Se}_{2}$ our results obtained within $\mathrm{LDA}^{\prime}+\mathrm{DMFT}$ approach were shown to be in better agreement wit experimental ARPES spectra, we have used the same $\mathrm{LDA}^{\prime}+\mathrm{DMFT}$ approach in our calculations for other doping levels. It was shown, that the form and position of the same quasiparticle bands is significantly transformed under doping. Position of quasiparticle bands with respect to each other also changes. Thus, depending on the doping level the role of correlation effects changes for each of the quasiparticle bands. To determine the influence of local Coulomb interaction upon each of Fe-3d bands we have calculated correlation renormalization factors and the energy shifts of $\mathrm{LDA}^{\prime}$ bands to match the maxima of spectral functions obtained in $\mathrm{LDA}^{\prime}+\mathrm{DMFT}$ calculations for all four dop- 
ing levels. Renormalization factors and energy shifts

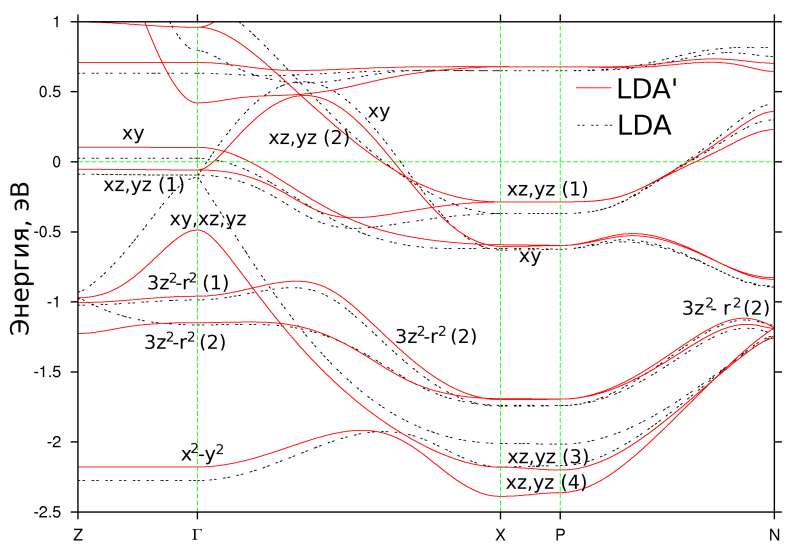

Fig. 4. Energy dispersions from $\mathrm{LDA}^{\prime}$ calculations (red lines) in $\mathrm{KFe}_{2} \mathrm{Se}_{2}$ along symmetry directions in the Brillouin zone with notations for those bands, for which renormalization factors were fitted. Black lines - energy dispersions from LDA calculations. Zero energy corresponds to the Fermi level.

(in parentheses) for $\mathrm{LDA}^{\prime}$ bands are listed in Table 1. Energy shifts are given for LDA' bands in electronvolts.

Black dotted lines in Fig. 4 represent LDA energy dispersions, which both in form and position are similar to energy dispersions obtained in $\mathrm{LDA}^{\prime}$ calculations. Thus, renormalization factors in $\mathrm{LDA}+\mathrm{DMFT}$ will be approximately the same as in $\mathrm{LDA}^{\prime}+\mathrm{DMFT}$ calculations (Table 1). Results for $\mathrm{K}_{0.76} \mathrm{Fe}_{1.72} \mathrm{Se}_{2}\left(\mathrm{n}_{e}=26.52\right)$ composition confirm this conclusion.

Energy dispersions of $\mathrm{LDA}^{\prime}$ calculation in the interval $(-1.0,0.4) \mathrm{eV}$ are stronger renormalized by iteractions, than energy dispersions below $-1.0 \mathrm{eV}$. Correlation renormalization factors for $3 z^{2}-r^{2}(2)$, xz, yz (3), xz, yz (4) and $\mathrm{x}^{2}-\mathrm{y}^{2}$ bands (Fig. 4 and Table 1), which in $\mathrm{LDA}^{\prime}$ calculations are below $-1.0 \mathrm{eV}$, are close to renormalization factor of of $\mathrm{Fe}-3 \mathrm{~d}$ band as a whole. Similarly, the xz, yz, xy, band, which belong to energy interval $(-1.0,-0.5) \mathrm{eV}$, is weakly renormalized as the whole Fe-3d band and its correlation renormalization factor does not depend on doping. The values of correlation renormalization for $\mathrm{xz}, \mathrm{yz}$ (2) band is weakly dependent on doping and is approximately equal to 2.5 , despite the fact, that this band is rather close to the Fermi level. Renormalization factor for $3 z^{2}-r^{2}$ (1) band increases sharply up to the value of 4.7 for filling $\mathrm{n}_{e}=26.52$.

For xz, yz (1) band the value of correlation renormalization in the lower Hubbard band monotonously grows with doping, while in the upper Hubbard band it monotonously decreases. For xy band dependence on doping is nonmonotonous. In its lower Hubbard band correlation effects grow with hole doping and reach maximum at $\mathrm{n}_{e}=27.20$, then decrease. In upper Hubbard xy band for $\mathrm{n}_{e}=29.00$ and $\mathrm{n}_{e}=27.20$ the value of correlation renormalization is large enough - about 4.0, while for $\mathrm{n}_{e}=28.00$ it is smaller -1.7 , and for $\mathrm{n}_{e}=26.52$ the upper Hubbard xy band is absent.

Thus, for $\mathrm{K}_{1-x} \mathrm{Fe}_{2-y} \mathrm{Se}_{2}$ compound the hole doping from stoichiometric composition $\mathrm{KFe}_{2} \mathrm{Se}_{2}$ up to $\mathrm{K}_{0.76} \mathrm{Fe}_{1.72} \mathrm{Se}_{2}$ leads to the growth of correlation effects for the same values of direct (Hubbard) $U$ and exchange (Hund) $J$ interactions. These effects are different for different quasiparticle bands and in different parts of the Brillouin zone.

Possibly the main expression of more strong role of correlation effects in this system, in contrast to previously studied iron based superconductors, is the absence of well-defined quasiparticles bands in the vicinity of the Fermi level.

\section{NEW SUPERCONDUCTORS - CHEMICAL ANALOGS OF IRON PNICTIDES}

\section{$3.1 \quad \mathrm{SrPt}_{2} \mathbf{A s}_{2}$ system}

Crystal structure.

In Ref. [46] it was shown, that $\mathrm{SrPt}_{2} \mathrm{As}_{2}$ has orthorhombic structure with space symmetry group Pmmn. In some sense this system reminds tetragonal crystal structure of $\mathrm{CaBe}_{2} \mathrm{Ge}_{2}$-type with space symmetry group $P 4 / \mathrm{nmm}$. We show this structure in Fig. 5. There are two layers of $\mathrm{PtAs}_{4}$ and $\mathrm{AsPt}_{4}$ tetrahedra in elementary cell. In one of these layers square lattice is formed by platinum ions, while in another by arsenic. Corresponding tetrahedrons are shown in Fig. 5 by lines. From the point of view of chemical formula $\mathrm{SrPt}_{2} \mathrm{As}_{2}$ is similar to HTSC system $\mathrm{BaFe}_{2} \mathrm{As}_{2}$ [47]. However, in the elementary cell of this last case there are two mirror reflected layers of $\mathrm{FeAs}_{4}$ tetrahedrons [24]. Note also, that majority of iron pnictides have $P 4 / \mathrm{nmm}$ structure [40]. In our calculations we have used an idealized tetrahedral structure with $P 4 / \mathrm{nmm}$ group (cf. details in Ref. [19]). Parameters of tetrahedral crystal structure can be determined averaging $a=4.482 \AA$ and $b=4.525 \AA$ for orthorhombic phase and taking $c=9.869 \AA$.

Electronic structure.

In Fig. 6 we show electronic bands calculated in LDA along directions of high symmetry in the first Brillouin zone of $\mathrm{SrPt}_{2} \mathrm{As}_{2}$. Note that $\mathrm{SrPt}_{2} \mathrm{As}_{2}$ bands close to M-point have some similarity with 1111 FeAs system $[23,26]$. However, close to the Fermi level bands are completely different from those in 1111 and 122 sys- 
Table 1. The values of correlation renormalization factors for separate LDA' bands, deboted in Fig. 4 . In parentheses - energy shifts on the scale of $\mathrm{LDA}^{\prime}$ bands in $\mathrm{eV}$.

\begin{tabular}{|c|c|c|c|c|}
\hline $\begin{array}{c}\text { Orbital } \\
\text { nature }\end{array}$ & $\mathrm{n}_{e}=26.52$ & $\mathrm{n}_{e}=27.20$ & $\mathrm{n}_{e}=28.00$ & $\mathrm{n}_{e}=29.00$ \\
\hline $\mathrm{xy} \mathrm{LHB}$ & $1.5(-0.23)$ & $3.9(-0.73)$ & $2.65(-0.61)$ & $1.7(-0.35)$ \\
$\mathrm{xy}$ UHB & no & $\sim 4.0(+0.60)$ & $\begin{array}{c}1.7(+0.25) \\
\text { part (top) }\end{array}$ & $\sim 4.0(+0.75)$ \\
\hline $\mathrm{xz}, \mathrm{yz}(1) \mathrm{LHB}$ & $4.2(-0.78)$ & $3.0(-0.75)$ & $2.6(-0.69)$ & $1.7(-0.38)$ \\
$\mathrm{xz}, \mathrm{yz}(1) \mathrm{UHB}$ & $1.6(+0.19)$ & $\sim 2.5(+0.48)$ & $3.0(+0.56)$ & $4.0(+0.77)$ \\
\hline $\mathrm{xz}, \mathrm{yz}(2)$ & $2.3(-0.48)$ & $\sim 2.5(-0.60)$ & $2.6(-0.69)$ & $1.7(-0.38)$ \\
\hline $\mathrm{xz}, \mathrm{yz}, \mathrm{xy}$ & $1.2(-0.1)$ & $1.3(-0.09)$ & $1.3(-0.10)$ & $1.4(-0.17)$ \\
\hline $3 \mathrm{z}^{2}-\mathrm{r}^{2}(1)$ & $4.7(-0.85)$ & $2.0(-0.30)$ & $1.3(-0.03)$ & $1.25(0.00)$ \\
$3 \mathrm{z}^{2}-\mathrm{r}^{2}(2)$ & $1.1(+0.25)$ & $1.3(0.00)$ & $1.3(-0.03)$ & $1.25(0.00)$ \\
\hline $\mathrm{xz}, \mathrm{yz}(3)$ & $1.1(+0.10)$ & $1.1(+0.17)$ & $1.0(+0.40)$ & $1.4(-0.10)$ \\
$\mathrm{xz}, \mathrm{yz}(4)$ & $1.1(+0.10)$ & $1.1(+0.15)$ & $1.0(+0.35)$ & $1.4(-0.17)$ \\
\hline $\mathrm{x}^{2}-\mathrm{y}^{2}$ & $1.1(+0.20)$ & $1.0(+0.32)$ & $1.3(-0.07)$ & $1.3(-0.07)$ \\
\hline $\mathrm{Fe} 3 \mathrm{~d}$ band & 1.3 & 1.3 & 1.25 & 1.25 \\
$\mathrm{as} \mathrm{a} \mathrm{whole}$ & & & & \\
\hline
\end{tabular}

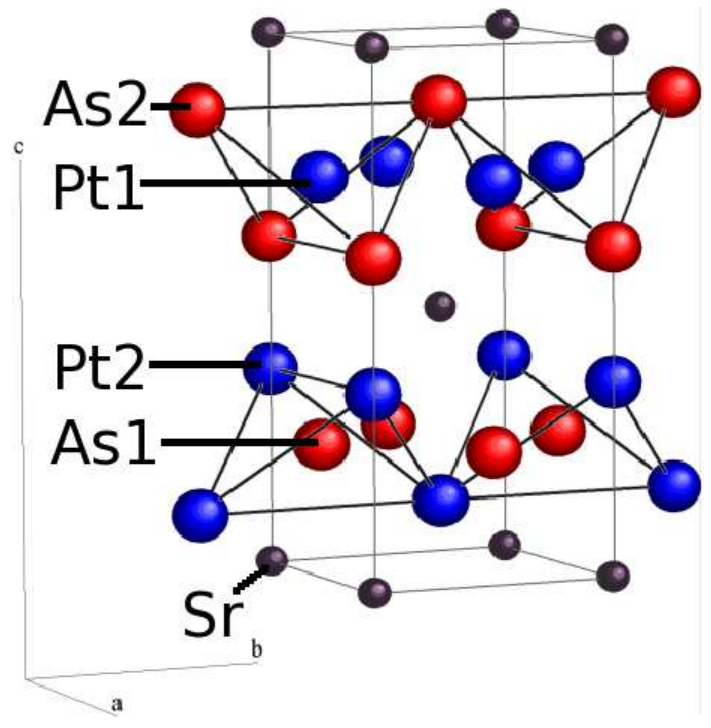

Fig. 5. Idealized tetragonal crystal structure of $\mathrm{SrPt}_{2} \mathrm{As}_{2}$.

tems. As in $\mathrm{SrPt}_{2} \mathrm{As}_{2}$ there are several bands crossing the Fermi level, we can speak about essentially multipleband nature of superconductivity in this system: four bands cross Fermi level in $\Gamma$-X direction and six in $\mathrm{M}-\Gamma$ direction.

As was shown in Ref. [19] the dominant contribution at the Fermi level is from Pt1-5d states. However, the joint contribution of Pt2-5d and As1-4p, As2-4p states

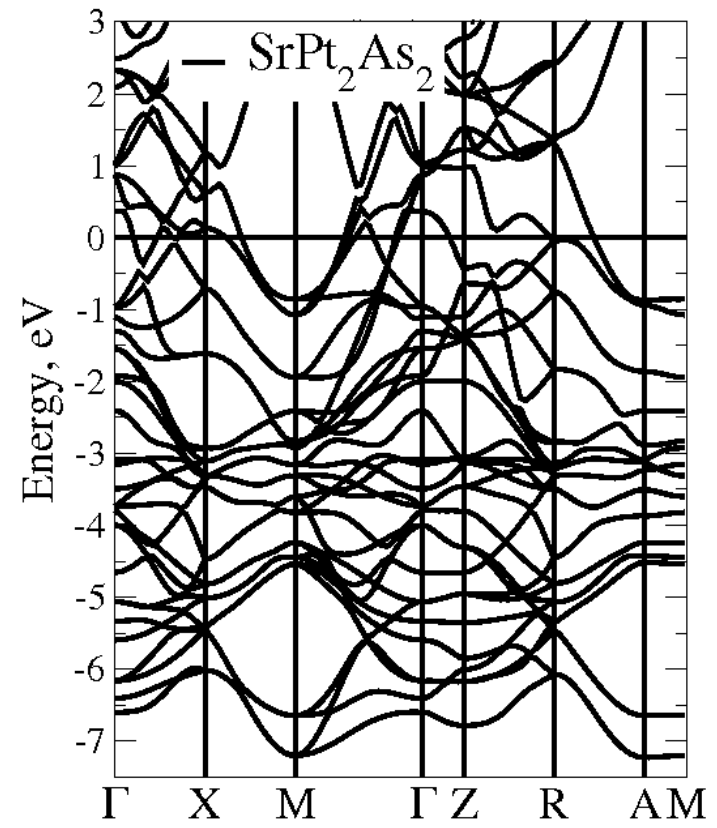

Fig. 6. LDA electronic dispersions for $\mathrm{SrPt}_{2} \mathrm{As}_{2}$. Zero energy at the Fermi level.

to the density of states at the Fermi level $E_{F}$ is also significant enough. This fact makes $\mathrm{SrPt}_{2} \mathrm{As}_{2}$ different from HTSC iron pnictides, where As-4p states are practically absent at the Fermi level $[23,24,25,26]$. The main part of the spectral weight of Pt-5d states is situ- 
ated well below the Fermi level, which is connected with larger number of valence electrons in $\mathrm{Pt}$, as compared to Fe. Obviously, Pt-5d states are more extended and produce wider band as compared to Fe-3d.

In Fig. 7 we show the complex picture of the Fermi surface for this system, obtained in LDA approximation. The sheets of this Fermi surface are essentially three-dimensional, which sharply differs $\mathrm{SrPt}_{2} \mathrm{As}_{2}$ from 1111 and 122 pnictides.

Thus we see, that $\mathrm{SrPt}_{2} \mathrm{As}_{2}$ has a complex band structure close to the Fermi level and complicated multisheet topology of the Fermi surface, which strongly differs from those observed in iron pnictides. In general case, the multiple-band system can support very complicated types of Cooper pairing with superconducting gaps of different sizes on different sheets of the Fermi surface, e.g. as in FeAs compounds [29, 30]. From general symmetry analysis [48, 49] it is known, that for tetragonal symmetry and singlet Cooper pairs either isotropic or anisotropic $s$-wave pairing is possible, as well as several types of $d$-wave pairing. However, from symmetry considerations only nothing can be said about $s^{ \pm}$-type of pairing with isotropic gaps of different signs on different sheets of the Fermi surface, which is considered to be most probable for iron pnictides $[2,30]$.

Let us make simplest estimates of the $T_{c}$ value within BCS theory. The value of density of states at the Fermi level $\mathrm{N}\left(E_{F}\right)$, obtained in our calculations is equal to 5.6 states $/ \mathrm{eV} /$ cell. Then the coefficient of linear temperature contribution to specific heat following from the results of LDA calculations will be $\gamma_{b}=\frac{\pi^{2}}{3} N\left(E_{F}\right)=13.1$ $\mathrm{mJ} / \mathrm{mole} / \mathrm{K}^{2}$, which is in reasonable agreement with experimental estimates giving $9.7 \mathrm{~mJ} / \mathrm{mole} / \mathrm{K}^{2}$ [8]. Let us estimate the pairing coupling constant $\lambda$ in BCS expression for $T_{c}=1.14 \omega_{D} e^{-1 / \lambda}$, using the experimental value of Debye frequency $\omega_{D}$. For $\omega_{D}=200 \mathrm{~K}$ and $T_{c}=5.2 \mathrm{~K}[8]$ we get for $\mathrm{SrPt}_{2} \mathrm{As}_{2} \lambda=0.26$. Then we can estimate $T_{c}$ for isovalent superconducting systems $\mathrm{BaNi}_{2} \mathrm{As}_{2}$ [6] and $\mathrm{SrNi}_{2} \mathrm{As}_{2}$ [7] with $T_{c}$ equal to $0.7 \mathrm{~K}$ [6] and $0.62 \mathrm{~K}$ [7] respectively. In this case we need the values of total LDA densities of states for BaNi and SrNi systems. Our calculations have given $\mathrm{N}\left(E_{F}\right)=3.86$ states $/ \mathrm{eV} /$ cell for $\mathrm{BaNi}$ and $\mathrm{N}\left(E_{F}\right)=2.81$ states $/ \mathrm{eV} /$ cell for $\mathrm{SrNi}$ system, which agree well with other calculations [50, 51]. Changing the value of $\lambda$ proportionally to the change of the value of $\mathrm{N}\left(E_{F}\right)$ we get $T_{c}$ for these systems equal to $0.97 \mathrm{~K}$ and $0.13 \mathrm{~K}$ respectively, in good agreement with experiments $[6,7]$.

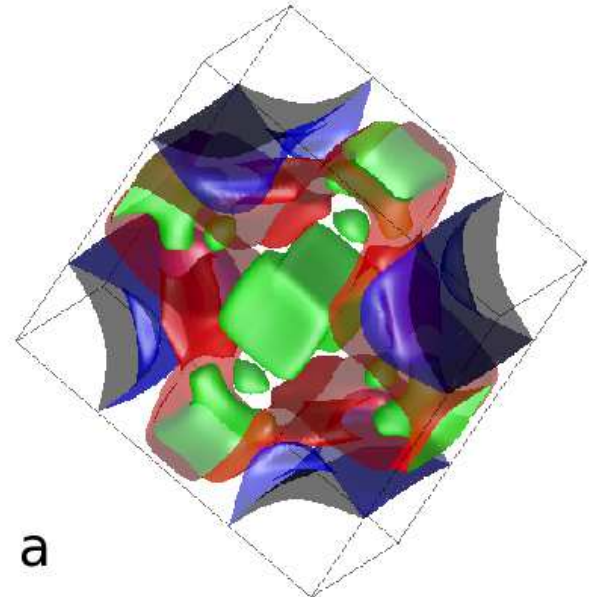

7. Fermi surface of $\mathrm{SrPt}_{2} \mathrm{As}_{2}$ obtained from LDA calculations.

\section{$32 \quad \mathbf{A P t}_{3} \mathbf{P}$ system}

Another new platinum based system - $\mathrm{APt}_{3} \mathrm{P}$ ( $\mathrm{A}=\mathrm{Sr}, \mathrm{Ca}, \mathrm{La})$ was discovered and described in Ref. [10], where the values of $T_{c}$ were obtained to be $8.4 \mathrm{~K}, 6.6 \mathrm{~K}$ and $1.5 \mathrm{~K}$ respectively. In this section we consider the electronic structure of $\mathrm{SrPt}_{3} \mathrm{P}$ system, first obtained in Ref. [21].

Crystal structure.

Crystals of $\mathrm{SrPt}_{3} \mathrm{P}$ belong to tetragonal symmetry group $P 4 / n m m$ with $a=5.8094 \AA$ and $c=5.3833 \AA$ [10] (cf. Fig. 8). Between strontium ions layers sit "antiperovskite" $\mathrm{Pt}_{6} \mathrm{P}$ octahedra, with $\mathrm{Pt} 1$ ions in layers occupy 4e $(1 / 4,1 / 4,1 / 2)$ positions, while apex Pt2 ions occupy - 2c $(0,1 / 2,0.1409)$. Phosphorous inside octahedra also occupy 2c positions with $z=0.7226$. Note that these $\mathrm{Pt}_{6} \mathrm{P}$ octahedra are not ideal: distances from $\mathrm{PPt}_{2}$ layer to different apex Pt2 ions are different, while inlayer Pt1 ions form ideal square. In Fig. 8 it is clearly seen, that $\mathrm{Pt}_{6} \mathrm{P}$ octahedra, having the common facets, form twodimensional plane with square lattice. In the following we assume, that $\mathrm{LaPt}_{3} \mathrm{P}$ has the crystal structure identical to that of $\mathrm{SrPt}_{3} \mathrm{P}$.

\section{Electronic structure.}

At the Fermi level density of states is essentially determined by Pt1-5d states of Pt1 ions forming twodimensional square lattice. (cf. Fig. 8). Also at the Fermi level there is a small admixture of Pt2-5d and P-3p states.

The value of density of states at the Fermi level $N\left(E_{F}\right)$ in compounds with $\mathrm{Sr}$ and La is equal to 4.69 states/eV/cell and 3.77 states/eV/cell correspondingly. These values are comparable with those in HTSC pnictides with relatively high $T_{c}$ (cf. Ref. [40]). Then the coefficient of linear term in specific heat $\gamma_{b}$ is equal 


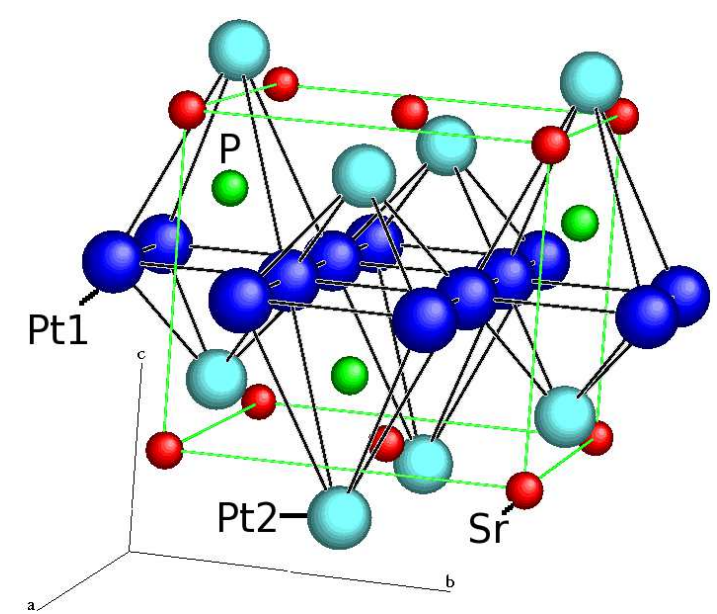

Fig. 8. Crystal structure of $\mathrm{SrPt}_{3} \mathrm{P} . \mathrm{Pt}_{6} \mathrm{P}$ octahedra are distinguished by $\mathrm{Pt}-\mathrm{Pt}$ bonds.

to $11 \mathrm{~mJ} / \mathrm{mole} / \mathrm{K}^{2}$ and $8.9 \mathrm{~mJ} / \mathrm{mole} / \mathrm{K}^{2}$ for $\operatorname{SrPt}_{3} \mathrm{P}$ and $\mathrm{LaPt}_{3} \mathrm{P}$ respectively, which agree rather well with experimental estimates for $\mathrm{SrPt}_{3} \mathrm{P}$ giving $\gamma^{\text {exp }}=12.7$ $\mathrm{mJ} / \mathrm{mole} / \mathrm{K}$ [10]. Strictly speaking $\gamma^{\exp }$ should be somehow larger, than those obtained in free-electron model, due to renormalization of the density of states by electron-phonon interaction: $\gamma=(1+\lambda) \gamma_{b}$, where $\lambda$ is dimensionless coupling constant. Comparing experimental and calculated values we can estimate this constant as $\lambda=0.15$, corresponding to weak coupling, which is insufficient to obtain the experimental value of $T_{c}$. Note, that according to experimental estimates made in Ref. [10], the so called Wilson ratio $R_{W} \sim 1$, which supports the absence of strong correlation effects in $\mathrm{SrPt}_{3} \mathrm{P}$.

In Fig. 9 we show band dispersions for $\operatorname{SrPt}_{3} \mathrm{P}$ (black lines) and $\mathrm{LaPt}_{3} \mathrm{P}$ (gray lines) in the vicinity of the Fermi level, obtained in LDA calculations. These dispersions strongly differ from dispersions in $\mathrm{SrPt}_{2} \mathrm{As}_{2}$ (cf. Fig. 6) [19], and from those in HTSC pnictides and chalcogenides (cf. Fig. 1) [5]. First of all, both $\mathrm{APt}_{3} \mathrm{P}$ systems are essentially three-dimensional, as can be seen from the presence of dispersion along $\Gamma-Z$ direction. From chemical composition point of view (neglecting the relaxation of lattice) the system $\mathrm{LaPt}_{3} \mathrm{P}$ looks like electron doped $\mathrm{SrPt}_{3} \mathrm{P}$ system.

Fig. 10 shows Fermi surfaces for $\operatorname{SrPt}_{3} \mathrm{P}$ (left) and $\mathrm{LaPt}_{3} \mathrm{P}$ (right) obtained by us via LDA calculations. In general, the form of Fermi surface of $\mathrm{APt}_{3} \mathrm{P}$ systems sharply differs from those in iron pnictides and chalcogenides $[24,27]$. In particular, these Fermi surfaces are essentially of three-dimensional nature and do not have well-defined cylinders.

From above discussion on electronic structure we can conclude, that $\mathrm{APt}_{3} \mathrm{P}$ compounds represent a new class

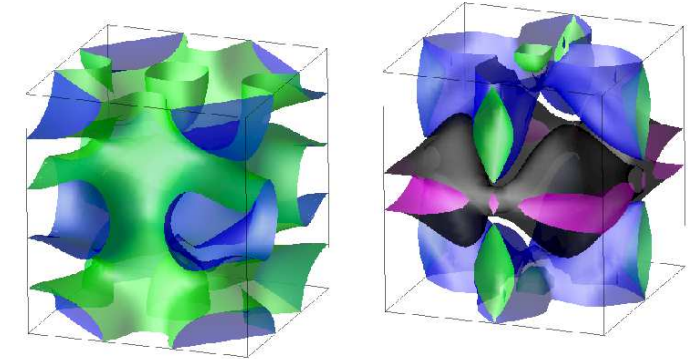

Fig. 10. Fermi surfaces for $\mathrm{SrPt}_{3} \mathrm{P}$ (left) and $\mathrm{LaPt}_{3}$ (right) obtained from LDA calculations.

of systems with multiple-band superconductivity as it was assumed in Ref. [10] from the measurements of Hall coefficient. $\mathrm{SrPt}_{3} \mathrm{P}$ is essentially a two-band superconductor, while $\mathrm{LaPt}_{3}$ possesses rather complicated band structure in the vicinity of the Fermi level. Fermi surfaces of both systems are characterized by large number of sheets with large number of "pockets" in the first Brillouin zone, with topology only slightly changing under doping.

In this case, from general symmetry analysis [48, 49] it is again cleat, that in case of singlet pairs, in principle, it is possible to have either the usual isotropic or anisotropic $s$-wave pairing or several types of $d$-wave pairing. Most probable is certainly the case of the usual $s$-wave pairing, as was assumed in Ref. [10]. Additional complications arise due to multiple-band nature of $\mathrm{APt}_{3} \mathrm{P}$ compounds. Three- dimensional structure of multisheet Fermi surface can lead to complicated structure of superconducting gap with different values of superconducting gaps on different sheets of the Fermi surface (cf. discussion if Refs. [29, 30]). However, the simple BCS expression for $T_{c}=1.14 \omega_{D} e^{-1 / \lambda}$ allows to estimate the dimensionless coupling constant $\lambda$ using the experimental values of $T_{c}$ and Debye frequency $\omega_{D}=190 \mathrm{~K}[10]$, which gives $\lambda=0.31$ for $T_{c}=8.4 \mathrm{~K}$. Lowering the value of $\lambda$ proportionally to the value of the density of states at the Fermi level, which changes from 4.69 states $/ \mathrm{eV} /$ cell in $\mathrm{SrPt}_{3} \mathrm{P}$ to 3.77 states $/ \mathrm{eV} /$ cell in $\mathrm{LaPt}_{3} \mathrm{P}$, we obtain $T_{c}=4 \mathrm{~K}$ for $\mathrm{LaPt}_{3} \mathrm{P}$, in reasonable agreement with experimental value of $1.5 \mathrm{~K}$.

Simplest BCS expression for $T_{c}$ gives only a rough estimate and it is better to use McMillan formula [52]. Rather close estimate can be also obtained from AllenDynes expression [53], which is the best interpolation formula for $T_{c}$ for strong coupling superconductors. Let us choose first an "optimistic" value of Coulomb pseudopotential $\mu^{*}=0$. Then McMillan's expression gives $\lambda=0.61$ for $\mathrm{SrPt}_{3} \mathrm{P}$ and, in turn, the value of $T_{c}=5.6 \mathrm{~K}$ for $\mathrm{LaPt}_{3} \mathrm{P}$. Assuming more typical value of $\mu^{*}=0.1$ 


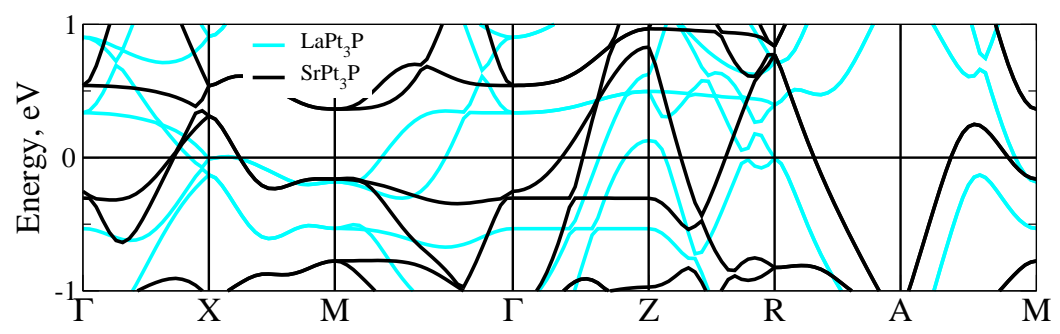

Fig. 9. LDA calculated electronic dispersions for $\mathrm{SrPt}_{3} \mathrm{P}$ close to the Fermi level (black lines) and $\mathrm{LaPt}_{3} \mathrm{P}$ (gray lines). Fermi level is at zero energy.

we obtain $\lambda=0.85$ for $\mathrm{SrPt}_{3} \mathrm{P}$, so that for $\mathrm{LaPt}_{3} \mathrm{P}$ we get $T_{c}=5.4 \mathrm{~K}$.

In summary, we can conclude, that the value of $T_{c}$ in $\mathrm{SrPt}_{3} \mathrm{P}$ and $\mathrm{LaPt}_{3} \mathrm{P}$ correlates rather well with the value of the total density of states at the Fermi level, similarly to the case of HTSC pnictides and chalcogenides $[5,40]$. At the same time, these estimates of $T_{c}$ correspond to weak or intermediate coupling superconductivity in $\mathrm{APt}_{3} \mathrm{P}$, which does not explain (cf. Ref. [53]) unusually high value of $2 \Delta / T_{c}$ ratio obtained from specific heat measurements in Ref. [10], hinting to the necessity of additional experimental verification of the value of $2 \Delta / T_{c}$ in these systems.

\section{$33 \quad \mathrm{BaFe}_{2} \mathrm{Se}_{3}$ system}

$\mathrm{BaFe}_{2} \mathrm{Se}_{3}$ (Ba123) system [11] was synthesized as a possible superconductor, analogous to $(\mathrm{K}, \mathrm{Cs}) \mathrm{Fe}_{2} \mathrm{Se}_{2}$ (with $T_{c} \sim 11 \mathrm{~K}$ according to preliminary data), though in a later work [12] superconductivity has not been observed up to $1.8 \mathrm{~K}$. In both works neutron diffraction was used to discover antiferromagnetic "spin ladders" with Neel temperature of the order of 250 , though the magnetic structure was not determined unambiguously. In this section we describe both electronic and magnetic structures of Ba123, obtained from LDA and LSDA calculations in Ref. [20].

Crystal structure.

We start as usual from description of crystal structure of Ba123. Ba123 system possesses orthorhombic symmetry group Pnma [11]. Basic structure elements in this compound are the so called "two-leg" ladders oriented along $b$-axis. These ladders are formed by iron ions, surrounded by tetrahedra formed by Se ions In orthogonal direction to. $b$-axis these ladders are placed in chessboard order (cf. Ref. [20]). Obviously, this crystal structure is sharply different from crystal structures of iron based HTSC systems (cf. Section 2 and Refs. $[5,24,27])$, which belong to primitive or body-centered tetragonal symmetry group.

Electronic structure.

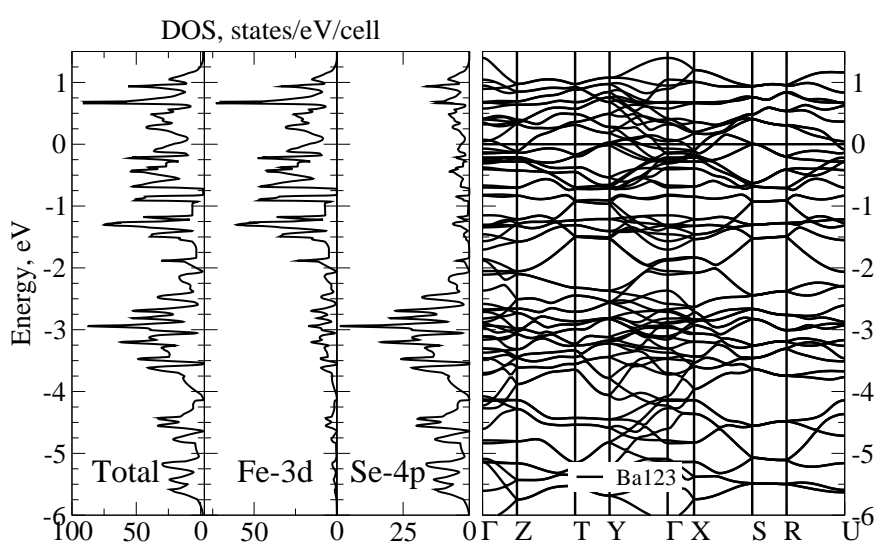

Fig. 11. Band dispersions and densities of states for Ba123 compound, obtained in LDA calculations. Fermi level - zero energy.

In Fig. 11 we show band dispersions (right) and densities of states (left) for Ba123 system Similarly to Ba122 systems [24] and $\mathrm{AFe}_{2} \mathrm{Se}_{2}$ [27] electronic states at the Fermi level are formed mainly by Fe-3d orbitals. Se- $4 \mathrm{p}$ orbitals form bands placed $-2 \mathrm{eV}$ lower in energy. Hybridization between Fe-3d and Se-4p states is relatively moderate.

Electronic bands of Ba123 system in close vicinity of the Fermi level strongly differ from those in iron pnictides and chalcogenides. [24, 27]. Around $\Gamma$-point we have two electronic pockets, while at the Brillouin zone edges (Y-point) there are three hole pockets (cf. Fig. 12). Close to the Fermi level we observe several Van-Hove singularities, making this system similar to $\mathrm{AFe}_{2} \mathrm{Se}_{2}$ (cf. Section 2) [27] and allowing changing Fermi surface topology by doping.

Fermi surface following from LDA calculations is shown in Fig. 12. The general form of this Fermi surface is completely different from the case of iron pnictides and chalcogenides [24, 27]. In Ba123 it is essentially three-dimensional and without explicit cylinders

Magnetic structure.

Neutron scattering experiments $[11,12]$ allowed to 


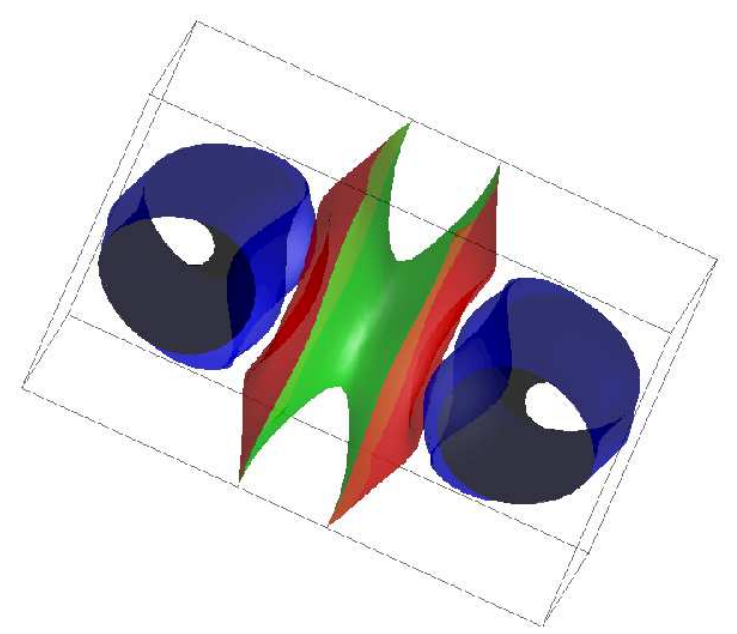

Fig. 12. Fermi surface of Ba123 obtained in LDA calculations.

determine Neel temperature $T \ll T_{N}^{\exp } \sim 250 \mathrm{~K}$ in Ba123, as well as two possible spin configurations, corresponding to irreducible representations $\tau_{1}$ and $\tau_{2}$ of space symmetry group Pnma, which produce practically identical diffraction patterns. In Ref. [20] the problem of the type of magnetic structure was solved by direct calculation of Neel temperatures for different spin configurations in mean-field approximation for Heisenberg model. Parameters of Heisenberg model were calculated within LSDA [54]. Our calculations demonstrated, that spin ladder configuration $\tau_{1}$ ("plaquettes") has Neel temperature $T_{N}\left(\tau_{1}\right)=217 \mathrm{~K}$, while for $\tau_{2}$ ("zigzags") $T_{N}\left(\tau_{2}\right)=186 \mathrm{~K}$, which makes $\tau_{2}$ configuration more favorable energetically, in accordance wit experimental work [12] and LSDA calculations of total energy [55].

Thus, despite similar to iron based HTSC chemical composition, Ba123 system possesses essentially different crystal and electronic structure, while from the point of view of magnetism it is a "spin ladder". The question of superconductivity in this system remains open.

\section{$34 \quad \mathrm{APd}_{2} \mathrm{As}_{2}$ system}

This section is devoted to electronic structure of $(\mathrm{Sr}, \mathrm{Ca}) \mathrm{Pd}_{2} \mathrm{As}_{2}$ and $\mathrm{BaPd}_{2} \mathrm{As}_{2}$ systems [22], where superconductivity was discovered with $\mathrm{T}_{c} 0.92 \mathrm{~K}$ and $1.27 \mathrm{~K}$ respectively [13]. We also compare these systems with isovalent system $(\mathrm{Sr}, \mathrm{Ba}) \mathrm{Ni}_{2} \mathrm{As}_{2}[56,57,58]$.

Crystal structure.

Crystal structure of $\mathrm{SrPd}_{2} \mathrm{As}_{2}$ and $\mathrm{CaPd}_{2} \mathrm{As}_{2}$ belongs to tetragonal space symmetry group $\mathrm{I} / 4 \mathrm{mmm}$, similarly to $\mathrm{BaFe}_{2} \mathrm{As}_{2}$ system [47]. We show this struc- ture in the left part of Fig.13. We can see, that it is analogous to Ba122 system [24].

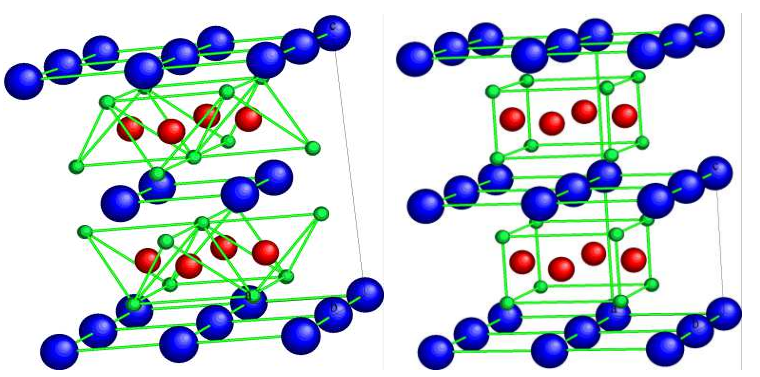

Fig. 13. Crystal structure of $(\mathrm{Sr}, \mathrm{Ca}) \mathrm{Pd}_{2} \mathrm{As}_{2}$ (left) and $\mathrm{BaPd}_{2} \mathrm{As}_{2}$ (right). Blue spheres - ions of $\mathrm{Sr}$ and $\mathrm{Ba}$, green $-\mathrm{As}$ and red $-\mathrm{Pd}$.

Though chemical formula of $\mathrm{BaPd}_{2} \mathrm{As}_{2}$ is similar to that of Ba122 pnictide, it has completely different crystal structure [13]. Space symmetry group of $\mathrm{BaPd}_{2} \mathrm{As}_{2}$ is $P / 4 \mathrm{mmm}$ and its structure is shown in right part of Fig. 13. This crystal structure, similarly to iron pnictides and chalcogenides, is a layered one, but Pd atoms do not possess surrounding tetrahedra of As atoms.

Electronic structure.

LDA calculated electronic dispersions along highsymmetry directions for $\mathrm{SrPd}_{2} \mathrm{As}_{2}$ and $\mathrm{BaPd}_{2} \mathrm{As}_{2}$, as well as densities of states, are shown in upper and lower parts of Fig. 14. From densities of states it can be seen, that in $\mathrm{SrPd}_{2} \mathrm{As}_{2}$ system, the main part of spectral weight is formed by $\mathrm{Pd}-4 \mathrm{~d}$ and $\mathrm{As}-4 \mathrm{p}$ states. $\mathrm{Pd}-4 \mathrm{~d}$ states belong to energy interval between -4 -0.5 (cf. upper part of Fig. 14), while As-4p states are in the energy interval (-6;-4) eV. As compared with Ba122 Pd-4d states are more extended in energy, than Fe-3d states. Also in $\mathrm{SrPd}_{2} \mathrm{As}_{2}$ we have considerable hybridization between $\mathrm{Pd}-4 \mathrm{~d}$ and As-4p states. The value of the density of states of $\mathrm{SrNi}_{2} \mathrm{As}_{2}$ at the Fermi level $N\left(E_{F}\right)=1.93$ states/eV/cell. which is twice lower, than in Ba122 system, due to the larger number of electrons in $\mathrm{Pd}$, as compared to Fe, leading to the shift of the Fermi level to the region of lower density of states.

In the lower part of Fig. 14 we show electronic dispersions and densities of states obtained for $\mathrm{BaPd}_{2} \mathrm{As}_{2}$ in LDA calculations. As the crystal structure of $\mathrm{BaPd}_{2} \mathrm{As}_{2}$ is completely different from that of $\mathrm{SrNi}_{2} \mathrm{As}_{2}$, it is of no surprise, that bands are also different. Note, that in Sr system, as well as in Ba system, the Fermi level is crossed by multiple bands, without any clear contributions from different valence shells. From LDA calculations we have found the value of total density of states at the Fermi level of $\mathrm{BaPd}_{2} \mathrm{As}_{2}$ to be 2.29 states $/ \mathrm{eV} /$ cell. 


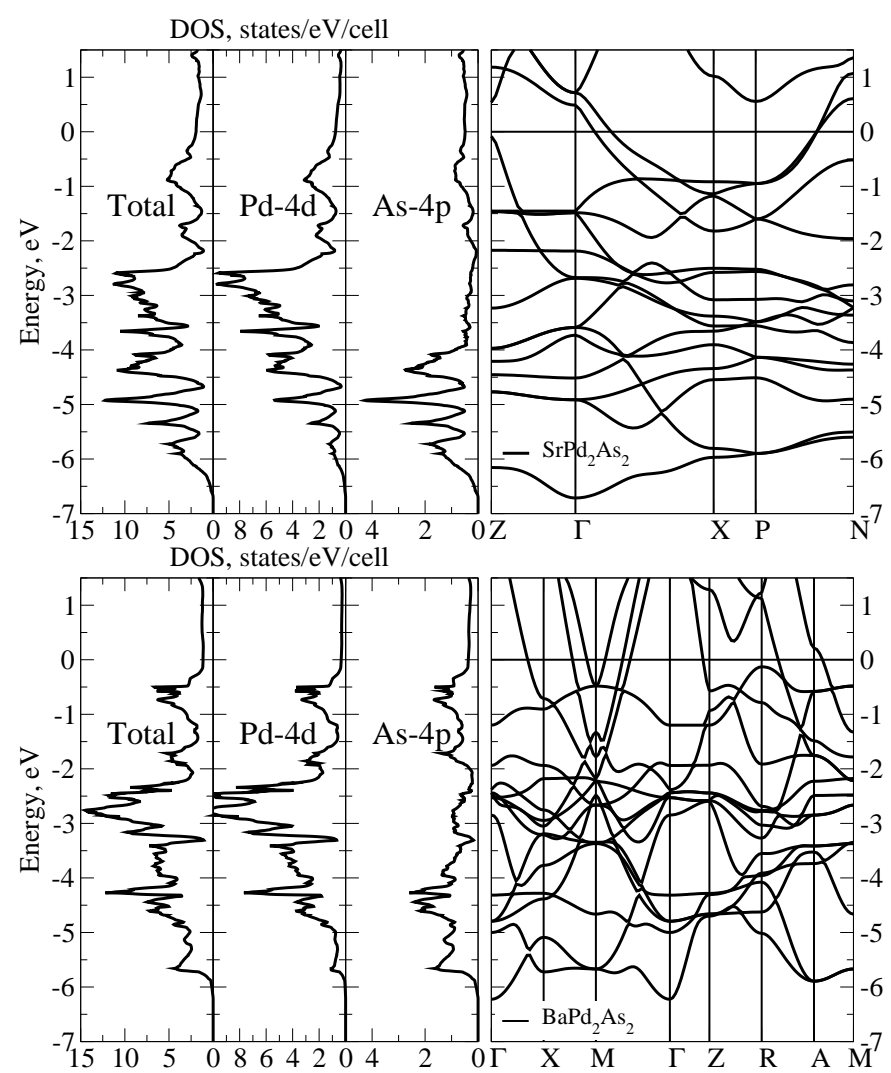

Fig. 14. Electronic dispersions and densities of states for $\mathrm{SrPd}_{2} \mathrm{As}_{2}$ (above) and $\mathrm{BaPd}_{2} \mathrm{As}_{2}$ (below) calculated in LDA. Fermi level - zero energy.

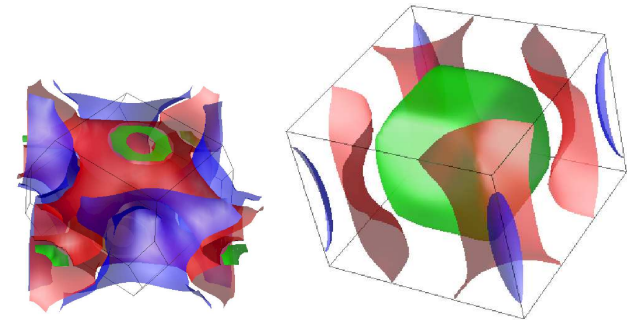

Fig. 15. LDA calculated Fermi surfaces for ( $\mathrm{Sr}, \mathrm{Ca}) \mathrm{Pd}_{2} \mathrm{As}_{2}$ (left) and $\mathrm{BaPd}_{2} \mathrm{As}_{2}$ (right).

In Fig. 15 we show Fermi surfaces for $\mathrm{SrPd}_{2} \mathrm{As}_{2}$ (left) and $\mathrm{BaPd}_{2} \mathrm{As}_{2}$ (right), obtained from our band structure calculations. Fermi surface for Sr system is essentially three-dimensional and complex, in contrast to Ba122 system [24], and consists of three sheets. Fermi surface of $\mathrm{BaPd}_{2} \mathrm{As}_{2}$, as compared with that of $\mathrm{SrPd}_{2} \mathrm{As}_{2}$, is rather simple, though also threedimensional, with hole-like sheet around $\Gamma$-point and electron=like sheets at the corners of Brillouin zone.

\section{CONCLUSION}

In this small review we considered electronic structure of a number of new superconductors, discovered during the experimental search of new candidate systems for high-temperature superconductivity, which followed the observation of HTSC in iron arsenides $[1,2,3,4]$. All these systems are multiple-band superconductors with rather complex topology of Fermi surfaces. From general theoretical considerations [30] it is clear, that multiple-band structure of electronic spectrum facilitates raising the temperature of superconducting phase transition $T_{c}$. However, the experimental picture is rather contradictory. In $\mathrm{K}_{1-x} \mathrm{Fe}_{2-y} \mathrm{Se}_{2}$ system superconductivity is observed at high enough temperatures, despite the absence of "nesting" of electronlike and hole-like Fermi surfaces, as well as the absence of well-defined quasiparticles close to the Fermi level (due to the strong role of electronic correlations in this systems). At the same time, the majority of other systems, considered above, demonstrate superconductivity at relatively low temperatures, despite clearly multipleband nature of electronic spectrum.

Because of this contradictory picture, the general question arises, whether there exists (or does not exist) some electronic structure, which is somehow "optimal" from the point of view of observation of HTSC. At present we can not give any definite answer to this question, though from our calculations it can be seen, that electronic spectra of the majority of systems with low $T_{c}$, considered above, have spectra sharply different from those in iron pnictide and iron chalcogenide HTSC systems. Attempts to find the general answer on the the posed question seem to be an interesting direction of further investigations.

This work is partially supported by RFBR grant 1402-00065, as well as by the grants of the programs of fundamental research of the Presidium of UB RAS "Quantum macrophysics and nonlinear dynamics" (projects No. 12-П-2-1002, 12-T-2-1001).

1. Y. Kamihara, T. Watanabe, M. Hirano, H. Hosono, J. Am. Chem. Soc. 130, 3296-3297 (2008).

2. M.V. Sadovskii, Uspekhi Fiz. Nauk 178, 1243 (2008); Physics Uspekhi 51, No. 12 (2008).

3. K. Ishida, Y. Nakai, H. Hosono. J.Phys. Soc. Jpn. 78, 062001 (2009).

4. P.J. Hirshfeld, M.M. Korshunov, I.I. Mazin. Rep. Prog. Phys. 74, 124508 (2011).

5. M.V. Sadovskii, E.Z. Kuchinskii, I.A. Nekrasov, JMMM 324 3481, (2012). 
6. E. D. Bauer, F. Ronning, B. L. Scott, and J. D. Thompson, Phys. Rev. B 78, 172504 (2008).

7. F. Ronning, N. Kurita, E.D. Bauer F. Ronning, N. Kurita, E.D. Bauer, B.L. Scott, T. Park, T. Klimczuk, R. Movshovich, J.D. Thompson, J. Phys. Condens. Matter, 20, 342203 (2008).

8. K. Kudo, Y. Nishikubo, M. Nohara, J. Phys. Soc. Jpn. 79, 123710 (2010).

9. S. Elgazzar, A.M. Strydom, S.-L. Drechsler, J. Supercond. Nov. Magn. 25, 1795 (2012).

10. T. Takayama, K. Kuwano, D. Hirai, Y. Katsura, A. Yamamoto, H. Takagi, Phys. Rev. Lett. 108, 237001 (2012).

11. A. Krzton-Maziopa, E. Pomjakushina, V. Pomjakushin, D. Sheptyakov, D. Chernyshov, V. Svitlyk, J.Phys.:Condens.Matter 23, 402201 (2011).

12. J. M. Caron, J. R. Neilson, D. C. Miller, A. Llobet, T. M. McQueen, Phys. Rev. B 84, R180409 (2011).

13. V.K. Anand, H. Kim, M.A. Tanatar, R. Prozorov, D.C. Johnston, Phys. Rev. B 87, 224510 (2013).

14. K. Held, I.A. Nekrasov, N. Blümer, V.I. Anisimov, D. Vollhardt, Int. J. Mod. Phys. B 15, 2611 (2001); G. Kotliar, S.Y. Savrasov, K. Haule, V.S. Oudovenko, O. Parcollet, C.A. Marianetti, Rev. Mod. Phys. 78, 865 (2006).

15. I.A. Nekrasov, N.S. Pavlov, M.V. Sadovskii, Pis'ma v ZhETF 95, 659 (2012) [JETP Letters 95, 581 (2012)].

16. I.A. Nekrasov, N.S. Pavlov, M.V. Sadovskii, 143, 713 (2013) [JETP 116, 620 (2013)].

17. I.A. Nekrasov, N.S. Pavlov, M.V. Sadovskii. Pis'ma v ZhETF 97, 18 (2013) [JETP Lett. 97, 15 (2013)].

18. I.A. Nekrasov, N.S. Pavlov, M.V. Sadovskii, 144, 1061 (2013) [JETP 117, 926 (2013)].

19. I.A. Nekrasov, M.V. Sadovskii Pis'ma v ZhETF 92, 833 (2010) [JETP Letters 92, 751 (2010)].

20. M.V. Medvedev, I.A. Nekrasov, M.V. Sadovskii, Pis'ma v ZhETF 95, 37 (2012) [JETP Letters, 95, 33 (2012)].

21. I.A. Nekrasov, M.V. Sadovskii, Pis'ma v ZhETF 96, 243 (2012) [JETP Letters 96, 227 (2012)].

22. I.A. Nekrasov, M.V. Sadovskii, Pis'ma v ZhETF 98, 28 (2013) [JETP Letters 98, 24 (2013)].

23. I.A. Nekrasov, Z.V. Pchelkina, M.V. Sadovskii, Pis'ma v ZhETF 87, 647 (2008) [JETP Letters 87, 560 (2008)].

24. I.A. Nekrasov, Z.V. Pchelkina, M.V. Sadovskii, Pis'ma v ZhETF 88, 155 (2008) [JETP Letters, 88, 144 (2008)].

25. I.A. Nekrasov, Z.V. Pchelkina, M.V. Sadovskii, Pis'ma v ZhETF 88, 621 (2008) [JETP Letters 88, 543 (2008)].

26. I.A. Nekrasov, Z.V. Pchelkina, M.V. Sadovskii, Pis'ma v ZhETF 88, 777 (2008) [JETP Letters 88, 679 (2008)].

27. I.A. Nekrasov, M.V. Sadovskii, Pis'ma v ZhETF 93, 182 (2011) [JETP Lett. 93, 166 (2011)].

28. O.K. Andersen. Phys. Rev. B 12, 3060 (1975); O. Gunnarsson, O. Jepsen, O.K. Andersen. Phys. Rev. B 27, 7144 (1983); O.K. Andersen, O. Jepsen. Phys. Rev. Lett. 53, 2571 (1984).
29. V. Barzykin, L.P. Gorkov. Pis'ma v ZhETF 88, 142 (2008) [JETP Letters 88, 131 (2008)].

30. E.Z. Kuchinskii, M.V. Sadovskii. Pis'ma v ZhETF 89, 176 (2009) [JETP Letters 89, 543 (2009)].

31. J. Guo, S. Jin, G. Wang, S. Wang, K. Zhu, T. Zhou, M. He, and X. Chen, Phys. Rev. B 82, 180520(R) (2010).

32. M. Fang, H. Wang, C. Dong, Z. Li, C. Feng, J. Chen, H.Q. Yuan, Phys. Rev. B 84, 224506 (2011).

33. W. Bao, Q. Huang, G. F. Chen, M. A. Green, D. M. Wang, J. B. He, X. Q. Wang, Y. Qiu, Chin. Phys. Lett. 28, 086104 (2011).

34. A.A.Kordyuk, Fiz. Nizk. Temp. 38, 1119 (2012).

35. H.-H.Wen, Rep. Prog. Phys. 75, 112501 (2012).

36. W.Li, H.Ding, Z.Li, P.Deng, K.Chang, K.He, S.Ji, L.Wang, X.Ma, J.-P.Hu, X.Chen, Q.-K.Xue, Phys. Rev. Lett., 109, 057003 (2012).

37. M.Yi, D.H.Lu, R.Yu, S.C.Riggs, J.-H.Chu, B.Lv, Z.K.Liu, M.Lu, Y.-T.Cui, M.Hashimoto, S.-K.Mo, Z.Hussain, C.W.Chu, I.R.Fisher, Q.Si, Z.-X.Shen, Phys. Rev. Lett., 110, 067003 (2013).

38. I.R. Shein, A.L. Ivanovskii, Phys. Lett. A 375, 1028 (2011).

39. Y. Mizuguhci, Y. Hara, K. Deguchi, S. Tsuda, T. Yamaguchi, K. Takeda, H. Kotegawa, H. Tou and Y. Takano, Supercond. Sci. Technol. 23, 054013 (2010).

40. E.Z. Kuchinskii, I.A. Nekrasov, M.V. Sadovskii, Pis'ma v ZhETF 91, 567 (2010) [JETP Letters, 91, 518 (2010)].

41. E.Z. Kuchinskii, I.A. Nekrasov, N.S. Pavlov, Zh. Eksp. Teor. Fiz. 144, 379 (2013); [JETP 117, 327 (2013)].

42. K. Haule, J.H. Shim and G. Kotliar, Phys. Rev. Lett. 100, 226402 (2008).

43. L. Craco, M.S. Laad, S. Leoni and H. Rosner, Phys. Rev. B 78, 134511 (2008).

44. A.O. Shorikov, M.A. Korotin, S.V. Streltsov, D.M. Korotin and V.I. Anisimov, 135, 134 (2009); [JETP 108, 121 (2008)]; arXiv: 0804.3283.

45. S. L. Skornyakov, A. V. Efremov, N. A. Skorikov, M. A. Korotin, Yu. A. Izyumov, V. I. Anisimov, A. V. Kozhevnikov, and D. Vollhardt, Phys. Rev. B 80, 092501 (2009).

46. A. Imre, A. Hellmann, G. Wenski, J. Graf, D. Johrendt und A. Mewisa, Z. Anorg. Allg. Chem. 633, 2037 (2007).

47. M. Rotter, M. Tegel, D. Johrendt. Phys. Rev. Lett. 101, 107006 (2008).

48. G.E. Volovik, L.P. Gor'kov. Zh. Eksp. Teor. Fiz. 88, 1412 (1985) [Sov. Phys. JETP 61, 843 (1985)]; Physica C 470, S418 (2010).

49. M. Siegrist, K. Ueda. Rev. Mod. Phys. 63, 239 (1991).

50. A. Subedi, D.J. Singh, Phys. Rev. B 78, 132511 (2008).

51. Z. G. Chen, G. Xu, W. Z. Hu, X. D. Zhang, P. Zheng, G. F. Chen, J. L. Luo, Z. Fang, and N. L. Wang, Phys. Rev. B 80, 094506 (2009).

52. W.L.McMillan, Phys. Rev. 167, 331 (1968).

53. P.B. Allen, R.C. Dynes, Phys. Rev. B 12, 905 (1975). 
54. A. I. Liechtenstein, M. I. Katsnelson, V. P. Antropov, V. A. Gubanov, J. Magn. Magn. Mater. 67, 65 (1987); V. I. Anisimov, F. Aryasetiawan, A. I. Lichtenstein, J. Phys.: Condens. Matter 9, 767 (1997).

55. B. Saparov, S. Calder, B. Sipos, H. Cao, S. Chi, D. J. Singh, A. D. Christianson, M. D. Lumsden, A. S. Sefat, Phys. Rev. B 84, 245132 (2011).

56. N. Kurita, F. Ronning, Y. Tokiwa, E. D. Bauer, A. Subedi, D. J. Singh, J. D. Thompson, R. Movshovich, Phys. Rev. Lett. 102, 147004 (2009).

57. Bo Zhou, Min Xu, Yan Zhang, Gang Xu, Cheng He, L. X. Yang, Fei Chen, B. P. Xie, Xiao-Yu Cui, M. Arita, K. Shimada, H. Namatame, M. Taniguchi, X. Dai, and D. L. Feng, Phys. Rev. B 83, 035110 (2011).

58. I.R. Shein, A.L. Ivanovskii, Physica B 405, 3213 (2010). 